\title{
Influence of pulsive pressure waves on liquid penetration into wood in semi-opened container
}

\author{
Soichi Tanaka ${ }^{1 *}$, Masako Seki ${ }^{2}$, Tsunehisa Miki ${ }^{2}$, Kenji Umemura ${ }^{1}$ and Kozo Kanayama ${ }^{1}$
}

\begin{abstract}
The purpose of this paper was to confirm the influence of pulsive pressure waves on the liquid penetration into wood in the semi-opened container. Wood block sample was irradiated by the pulsive pressure waves in the semi-opened container filled with water used as a liquid. The irradiation was also performed in the closed container for the comparison. The water penetration into the sample was promoted by the pressure-wave irradiation. There was little difference in the degree of the penetration between the closed and the semi-opened containers. It was presumed from the measured hydraulic pressure that the pressure-wave energy irradiated on the sample in the closed container was higher than that in the semi-opened container. It was also presumed that the cavitation generation was promoted in the semi-opened container. This indicates that the cavitation as well as the pressure waves themselves affected the liquid penetration into wood. The compressive deformation of the sample irradiated in the semi-opened container was slightly smaller than that in the closed container. This indicates that the pulsive pressure-wave irradiation in the semi-opened container promoted the liquid penetration into wood with less compressive deformation.
\end{abstract}

Keywords: Impregnation of wood, Pressure-wave treatment, Permeability of wood, Collapse of wood, Aspirated pit, Tyloses

\section{Introduction}

Impregnation of wood with liquid is one of the most important techniques to improve its disadvantages and to add new properties to it. For the effective impregnation, the liquid is required to be penetrated into wood so that the unpenetrated areas are as small as possible. The most popular impregnation is the pressure process, which is composed of immersing wood in the liquid under vacuumed pressure, exposing the liquid to an atmospheric pressure, and often placing it under pressurized pressure. In this process, the liquid penetrates wood mainly due to the liquid flow driven by higher hydraulic pressure at the wood surface than that at the tip of the penetration inside wood.

\footnotetext{
*Correspondence: soichi_tanaka@rish.kyoto-u.ac.jp

${ }^{1}$ Research Institute for Sustainable Humanosphere, Kyoto University, Uji,

Kyoto 611-0011, Japan

Full list of author information is available at the end of the article
}

The flow of the liquid is often disturbed by the discontinuous flow paths, which causes the unpenetrated areas remained in wood. The discontinuity also leads to the collapse of wood, because of the lower pressure required to deform wood than the pressure required for the liquid flow. The discontinuity is mainly due to the closed tissues such as the aspirated pits in softwood and the tyloses in hardwood (for example, [1]). Therefore, the treatment for decreasing the discontinuity by breaking the closed tissues has been studied in biological (for example, [2]), chemical (for example, [3]), or physical way (for example, [4]).

Pressure-wave treatment is one of the promising techniques for decreasing the discontinuity. This technique has an advantage of both decreasing the discontinuity and promoting the liquid penetration in one process. In the technique, the pressure waves are irradiated on wood through the surrounding liquid, causing the kinetic momentum of the liquid in wood. The impact of the momentum is expected to break the closed tissues, which 
can decrease the discontinuity. The previous studies have shown that the liquid penetration was promoted by irradiating the pressure waves such as ultrasonic waves (for example, [5]), sonic waves [6], and shockwaves [7].

The conventional pressure-wave treatments have several practical problems. The ultrasonic- and sonic-wave irradiations generally have a small effect on the liquid penetration, since these waves do not have much energy enough to affect deeply inside wood $[5,6]$. To enhance the effect, the sonic waves were irradiated on wood under the pressurized pressure in a closed pressure container [6], but the pressure container takes cost to ensure safety especially in legal perspective. Furthermore, the shockwave irradiation is dangerous though it may have a larger effect on the liquid penetration. This is because the explosives [7] or the electrical discharge [8] is used as a wave source. The pressure-wave treatment, therefore, will become more useful, if it has a large effect on the liquid penetration in a low-cost container with a safe wave source.

In this study, we proposed the pulsive pressure-wave irradiation on wood in a semi-opened container filled with liquid. The container was hit to oscillate the waves. This wave source was far safer than the explosives and the electrical discharge. The semi-opened container was used to enhance the safety in low cost compared to the closed container. Furthermore, in the semi-opened container, the liquid penetration is expected to be promoted by the cavitation in the liquid as well as the pressure waves themselves propagated in wood, detailed in "Materials and methods." The cavitation is known as the phenomenon, where the minute bubbles are grown under negative gauge pressure and are subsequently burst under increasing pressure to generate the impact [9]. The liquid penetration into wood can be promoted, if the cavitation bubbles were burst to add the impact on the closed tissues. In a previous study [5], the cavitation was suggested to promote the penetration, though it has not yet been well examined.

The purpose of this paper was to confirm the influence of the pulsive pressure waves on the liquid penetration into wood in the semi-opened container. The apparatus equipped with the semi-opened and closed containers was experimentally produced. The degree of the penetration, or the retention, after the irradiation of the pulsive pressure waves on a wood block sample was evaluated by using the closed or semi-opened container. The retention was discussed in terms of the pressure-wave energy and the cavitation, which was presumed using the pressure variation around wood. The compressive deformation of wood during the pressure-wave irradiation was also evaluated by comparing the dimension of the irradiated sample with that of the non-irradiated one.

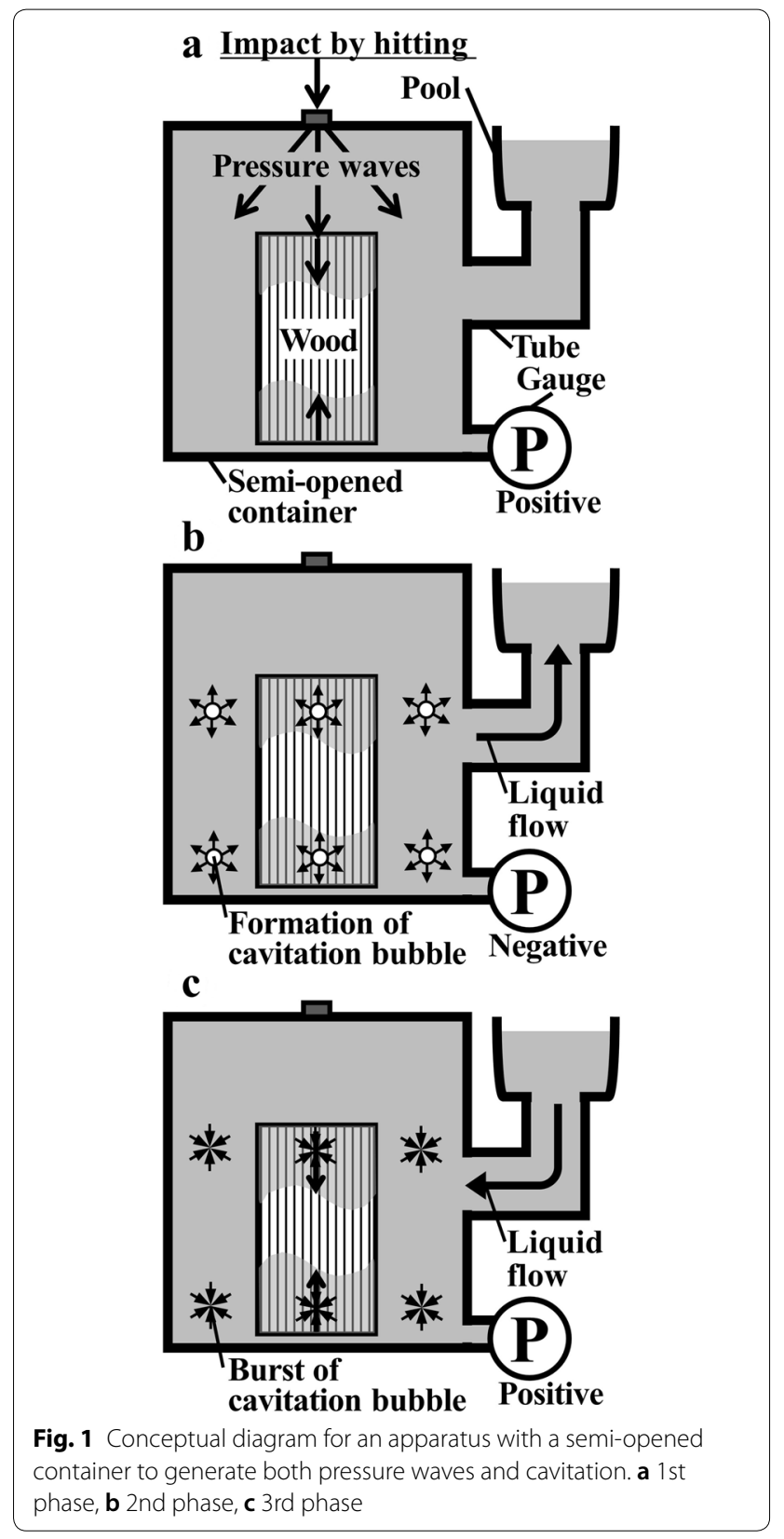

\section{Materials and methods}

Concept of an experimental system

Figure 1 shows the conceptual diagram for an apparatus composed of the semi-opened container with the pool, and the tube connecting them. All components are filled with the liquid which is assumed to be continuous from the wave source to the closed tissues in wood. The pulsive pressure waves are generated by hitting the container (Fig. 1a). A part of the waves must be propagated through the liquid into wood. This can cause the kinetic momentum in cell cavities to add the impact on the closed 


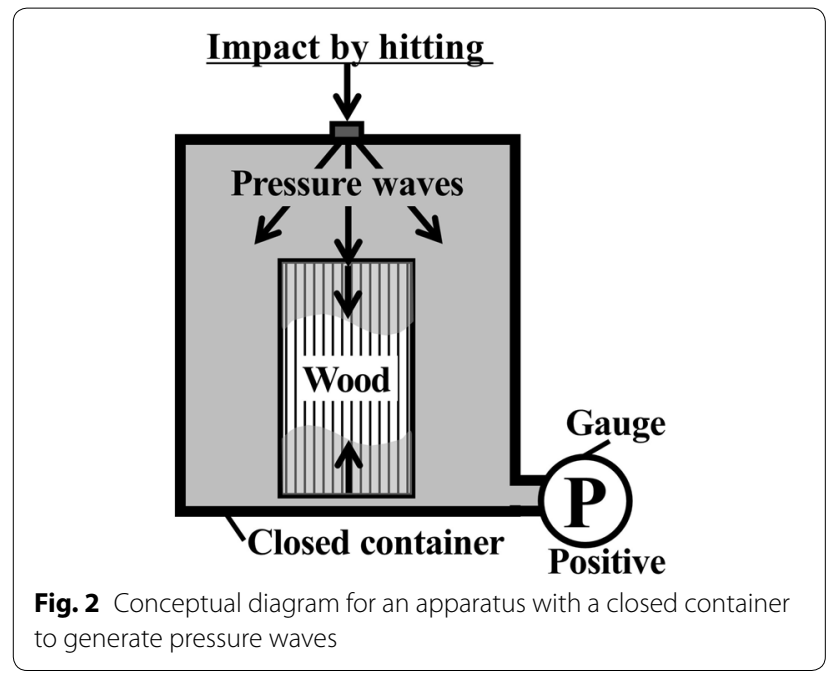

tissues. Another part of the waves must cause the liquid flow in the tube toward the pool. The kinetic momentum in the tube should make the hydraulic pressure in the container negative (Fig. 1b). Cavitation bubbles therefore can be formed in the liquid inside the container and the wood block. The lower pressure inside the container than the pressure outside should subsequently cause the kinetic momentum in the tube toward the container (Fig. 1c), causing the positive pressure in the container. Thus, the cavitation bubbles can burst to add the impact on the closed tissues. Only in this container, however, it was difficult to discuss the influence of the cavitation, since both the pressure waves themselves and the cavitation can promote the liquid penetration into wood. Thus, an apparatus with the closed container is also designed (Fig. 2). In this container, only the pressure waves can affect the liquid penetration. The influence of the cavitation will be revealed if the degree of the penetration for the semi-opened container (Fig. 1) is compared to that for the closed one (Fig. 2).

\section{Preparation of an apparatus and samples}

Figure 3 shows the experimental apparatus to irradiate the pulsive pressure waves on a wood sample in a closed or a semi-opened container. The container is capable of being separated into two pieces at the joint coupler so that the sample is placed on and taken out of the container. The fixture with sealant allows to fix the sample without the liquid leakage from the container. A feed pump has an adequate ability to supply the liquid to fill up the container. To realize a closed container, the valves $A, B$, and $C$ are closed. However, to realize a semiclosed container, only the valve $B$ is opened. To generate the impulsive pressure waves, the container filled with the liquid is fixed to the ground and hit by dropping a

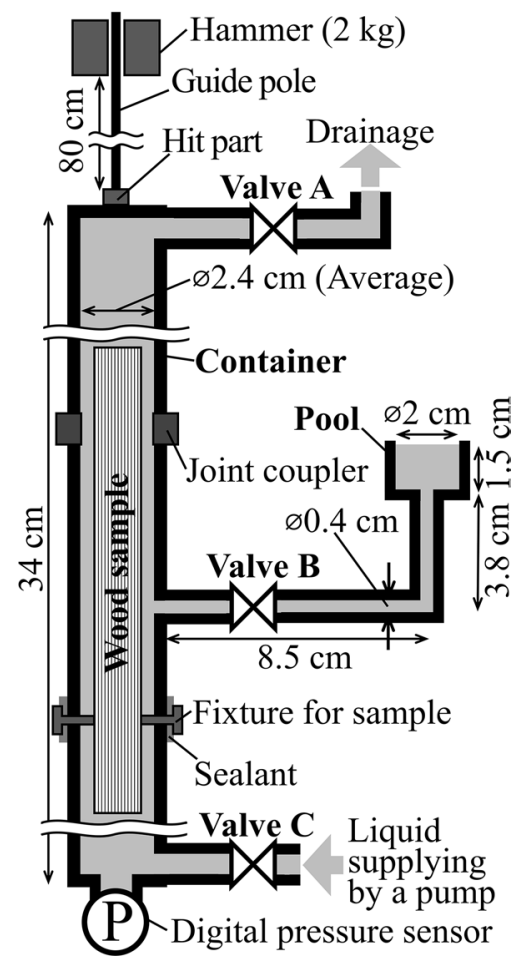

Fig. 3 Experimental apparatus

hammer $(2 \mathrm{~kg})$ along a guide pole from $80 \mathrm{~cm}$ height. The temporal variability of the hydraulic pressure in the container after hitting was measured by using a digital pressure sensor (GM-025, Keyence Co.) connected to a data collecting system (NR-500 and NR-HA08, Keyence Co.). The $90 \%$ response time of the sensor and the sampling interval of the system were $5 \mathrm{~ms}$ and $50 \mu \mathrm{s}$, respectively. The experimental apparatus was placed in a room with a temperature of $23-24{ }^{\circ} \mathrm{C}$. Distilled water at $22-23^{\circ} \mathrm{C}$ was used as the liquid.

The wood samples with $15 \mathrm{~mm} \quad(\mathrm{R}) \times 15 \mathrm{~mm}$ $(\mathrm{T}) \times 150 \mathrm{~mm}(\mathrm{~L})$ were prepared from the heartwood blocks of kiri (Paulownia tomentosa) and Alaska cedar (Chamaecyparis nootkatensis). The samples were dried at $105{ }^{\circ} \mathrm{C}$ to be an oven-dried condition. Mass and volume of the dried sample, $m_{0}$ and $v_{0}$, were measured.

\section{Experimental procedure}

The liquid was penetrated into a wood sample in three kinds of impregnation conditions: control and pressurewave irradiations in the closed and semi-opened containers. The oven-dry density and the number of test samples for each impregnation condition are summarized in Table 1.

The procedure in common with all the impregnation conditions was performed as the following. The dried 
Table 1 Oven-dry density and number of test samples for each treatment condition

\begin{tabular}{llcc}
\hline Species & Treatment condition & $\begin{array}{l}\text { Oven-dry density, } \boldsymbol{m}_{\mathbf{0}} / \mathbf{v}_{\mathbf{0}}(\mathbf{g} / \mathrm{c} \\
\mathbf{c m}^{\mathbf{3}} \text { ) }\end{array}$ & $\begin{array}{l}\text { Number } \\
\text { of test } \\
\text { samples, } \boldsymbol{n}\end{array}$ \\
\hline kiri & Control (no irradiation) & $0.274^{\mathrm{a}} \pm 0.010^{\mathrm{b}}$ & 4 \\
& Pressure-wave irradiation in a semi-opened container & $0.279 \pm 0.013$ & 4 \\
Alaska cedar & Pressure-wave irradiation in a closed container & $0.279 \pm 0.009$ & 4 \\
& Control (no irradiation) & $0.492 \pm 0.027$ & 6 \\
& Pressure-wave irradiation in a semi-opened container & $0.492 \pm 0.022$ & 6 \\
\hline
\end{tabular}

a Average

b Standard deviation

sample with the mass of $m_{0}$ was placed under a vacuumed pressure (An absolute pressure of $0.8 \mathrm{kPa}$ ) with the temperature of $23-24{ }^{\circ} \mathrm{C}$. An hour later, the sample was quickly immersed in the liquid under the vacuumed pressure. The start time of the immersion is defined at the origin of time, i.e., $t=0$. At $t=6 \mathrm{~s}$, the liquid that surrounded the sample was quickly exposed to an atmospheric pressure. The sample was maintained in the liquid for promoting the liquid injection into wood until $t=9 \mathrm{~min} 30 \mathrm{~s}$ so that the liquid could be continuous from the wood surface to the closed tissues. Mass of the liquid-injected sample, $m_{\mathrm{i}}$, was measured at $t=10 \mathrm{~min}$. The sample was placed in the apparatus (Fig. 3) with all valves closed, and the valves $A$ and $C$ were opened at $t=11 \mathrm{~min}$. The apparatus was fully filled with the liquid and the air in the apparatus was expelled by using the feed pump at $t=11 \mathrm{~min} 30 \mathrm{~s}$. The valves $\mathrm{A}$ and $\mathrm{B}$ were closed and opened, respectively, and the pool was filled with the liquid using the pump, and the valve $C$ was subsequently closed at $t=11 \mathrm{~min} 40 \mathrm{~s}$. The treatment for each impregnation condition was started at $t=11 \mathrm{~min} 57 \mathrm{~s}$ and finished at $t=42 \mathrm{~min}$. The sample was taken out of the apparatus at $t=42 \mathrm{~min}$, and mass of the treated sample, $m_{\mathrm{w}}$, was measured at $t=43 \mathrm{~min}$. The samples were subsequently immersed in the liquid at $t=43 \mathrm{~min} 30 \mathrm{~s}$. The immersion was maintained to obtain the maximum volume of the samples. For the kiri samples, the volume after the 3-day immersion was defined as the maximum volume, $v_{\mathrm{s}}$, since the moisture content (MC) reached to 248-280\%, which was far larger than the fiber saturation point (FSP). While for the Alaska cedar samples, the volume after the 5-day immersion was defined as $v_{\mathrm{s}}$, since it took 5 days for the MC to reach $85-104 \%$, being far larger than the FSP.

The treatment for each impregnation condition between $t=11 \min 57 \mathrm{~s}$ and $42 \mathrm{~min}$ was performed as the following. For pressure-wave irradiation in the semiopened container, the hitting of the container, which was started at $t=12 \mathrm{~min}$, was repeated 3 times at the interval of $20 \mathrm{~s}$. The 3-times repeated hittings were totally repeated 10 times at the intervals of $3 \mathrm{~min}$, say, the total number of the hittings was 30 . For pressure-wave irradiation in the closed container, the valve $\mathrm{B}$ was closed $3 \mathrm{~s}$ before every hitting and was opened $3 \mathrm{~s}$ after every hitting, while the procedure of the hitting itself was the same as that in the semi-closed container. For control, the container was not hit at all with the valve $B$ being opened.

\section{Definition of parameters}

The cavity filling rates before and after the treatment for each impregnation condition were defined as follows:

$$
\begin{aligned}
& \xi_{\mathrm{i}}=100\left(m_{\mathrm{i}}-m_{0}\right) /\left\{\rho_{\mathrm{l}}\left(v_{\mathrm{s}}-m_{0} / \rho_{\mathrm{c}}\right)\right\}[\%], \\
& \xi_{\mathrm{w}}=100\left(m_{\mathrm{w}}-m_{0}\right) /\left\{\rho_{\mathrm{l}}\left(v_{\mathrm{s}}-m_{0} / \rho_{\mathrm{c}}\right)\right\}[\%],
\end{aligned}
$$

where $\rho_{\mathrm{c}}$ represents oven-dry density of cell wall $(=1.5 \mathrm{~g} /$ $\left.\mathrm{cm}^{3}\right)$, and $\rho_{1}$ density of the liquid, or the distilled water $\left(=1.0 \mathrm{~g} / \mathrm{cm}^{3}\right)$. The value of $\xi_{\mathrm{i}}$ was evaluated as an indicator of the permeability unique to each wood sample. The value of $\xi_{\mathrm{w}}-\xi_{\mathrm{i}}$ was evaluated as an indicator of the liquid penetration during the treatment.

The maximum volumetric swelling $\beta_{\mathrm{m}}$ was defined as the following equation:

$$
\beta_{\mathrm{m}}=100\left(v_{\mathrm{s}}-v_{0}\right) / v_{0}[\%] \text {. }
$$

The compressive deformation was evaluated by comparing the value of $\beta_{\mathrm{m}}$ for the pressure-wave irradiation with that for the control.

\section{Results and discussion}

Cavity filling rate for the samples of kiri and Alaska cedar is shown in Figs. 4 and 5, respectively. The filling rate before the treatment, $\xi_{\text {i }}$, indicating the permeability unique to each sample, was distributed in the similar range for all treatment conditions. The change in the filling rate, $\xi_{\mathrm{w}}-\xi_{\mathrm{i}}$, indicating the liquid penetration during 


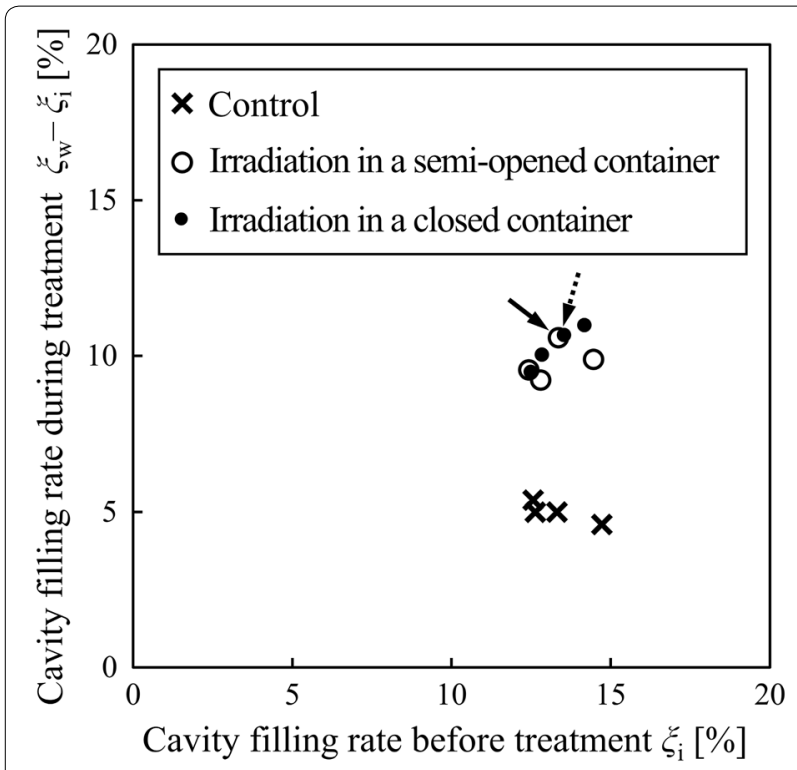

Fig. 4 Cavity filling rate for samples of kiri

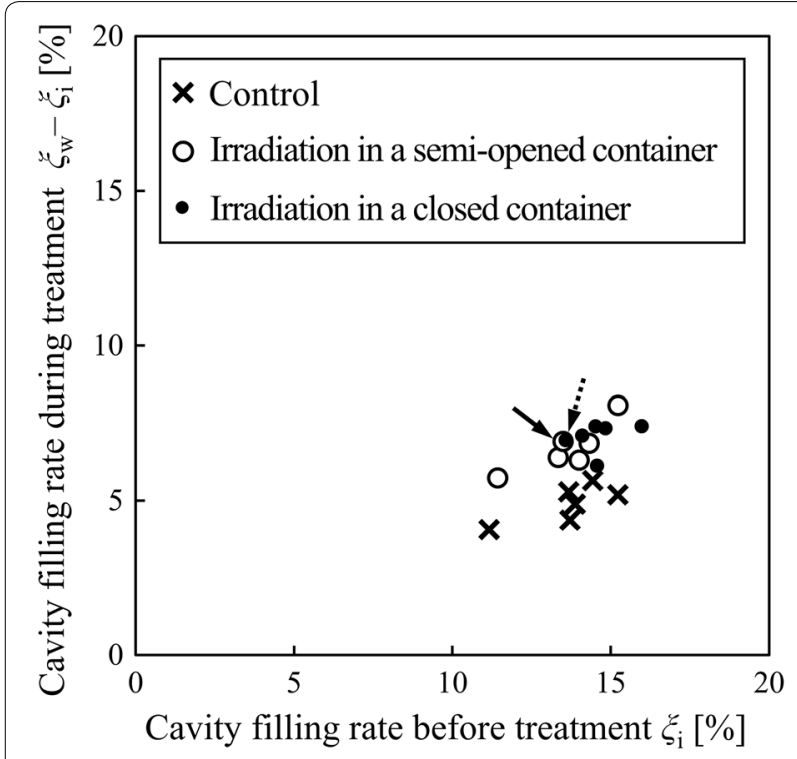

Fig. 5 Cavity filling rate for samples of Alaska cedar

the treatment, for the pressure-wave irradiations was larger than that for control in both species. The values of $\xi_{\mathrm{w}}-\xi_{\mathrm{i}}$ for the kiri samples (Fig. 4) were larger than those for the Alaska cedar ones (Fig. 5). This may be due to the thinner closed tissues for kiri than those for Alaska cedar. In Figs. 4 and 5 , the values of $\xi_{\mathrm{w}}-\xi_{\mathrm{i}}$ for the irradiation in the semi-opened container were distributed in the same range as those in the closed one. This indicates that there was little difference in the degree of the penetration

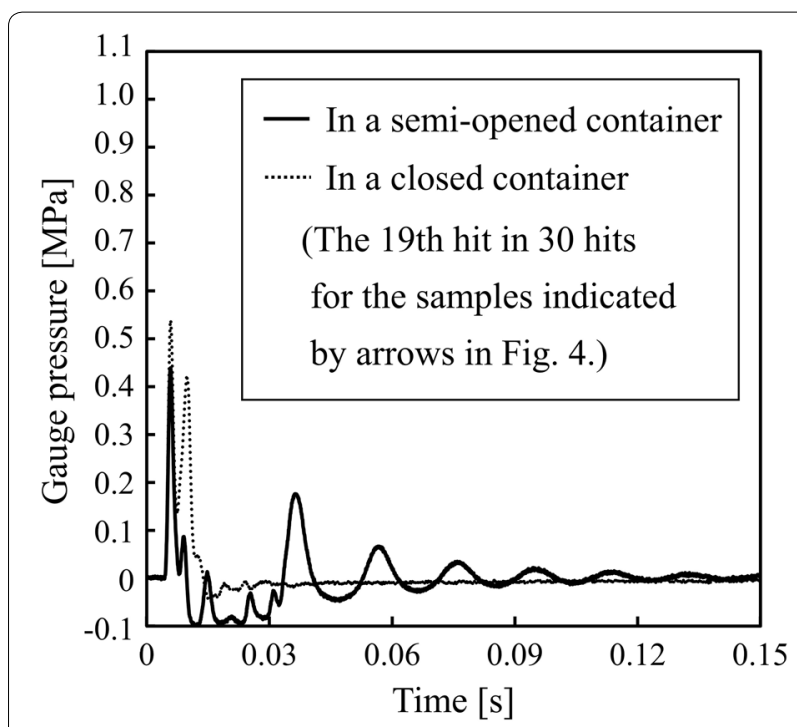

Fig. 6 Temporal variability soon after a generation of pressure waves for sample of kiri

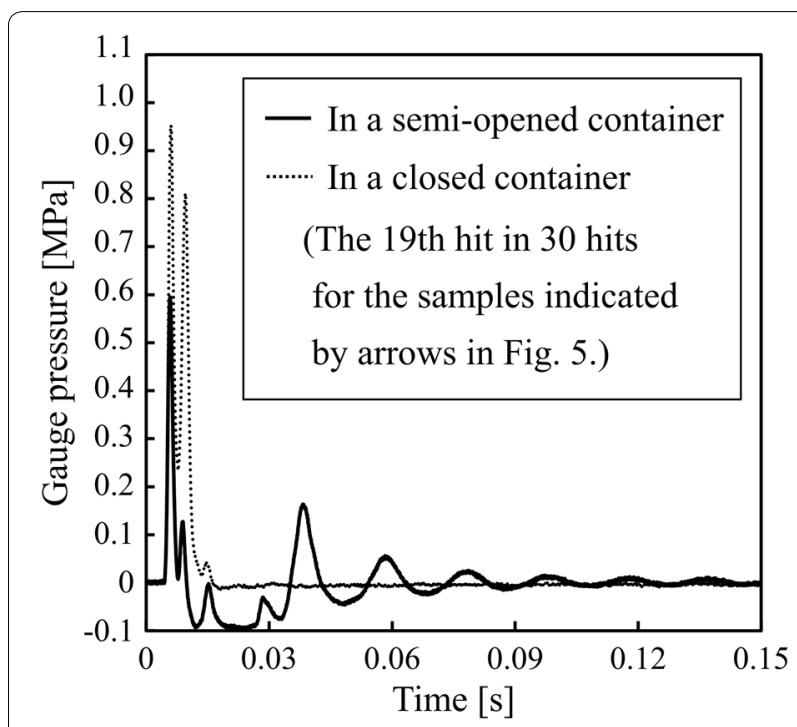

Fig. 7 Temporal variability soon after a generation of pressure waves for sample of Alaska cedar

between the closed and the semi-opened containers for both species.

The representative examples of temporal variability of hydraulic pressure in the container are shown in Figs. 6 and 7 . The maximum hydraulic pressure, implying the pressure-wave energy irradiated on the sample, for the Alaska cedar sample (Fig. 7) was larger than that for the kiri sample (Fig. 6). This may be due to the less air remained in the Alaska cedar sample than that in the kiri 
sample, leading to the less absorption of the pressurewave energy to the remained air.

The maximum hydraulic pressure for the closed container was larger than that for the semi-opened one for both wood species (Figs. 6 and 7). This was because the part of the liquid was able to be flown into the pool for the semi-opened container. Thus, the pressure-wave energy irradiated on the sample in the closed container was presumed to be higher than that in the semi-opened container. From this viewpoint, the liquid penetration in the closed container would be larger than that in the semi-opened one, because the pressure waves would add the larger impact on the closed tissues in the closed container. However, in Figs. 4 and 5, there was little difference in the degree of the penetration between the closed and the semi-opened containers for both species. To explain this inconsistency, the other factors except the pressure-wave energy should be taken into consideration. The cavitation is considered to be one of the largest factors. In the semi-opened container in Figs. 6 and 7, soon after the first increase in the pressure, the gauge pressure decreased to a negative value getting closer to $-0.1 \mathrm{MPa}$ with minimum absolute pressures at $2.6 \mathrm{kPa}$ and $0.6 \mathrm{kPa}$, respectively. The cavitation bubbles in water are known to be formed [9], if the pressure is smaller than water vapor pressure $\left(2.6-3.0 \mathrm{kPa}\right.$ at $22-24{ }^{\circ} \mathrm{C}$ [10]). Thus, the cavitation generation was presumed to be promoted in the semi-opened container. On the other hand, the gauge pressure in the closed container did not get closer to $-0.1 \mathrm{MPa}$. It was therefore presumed that the cavitation bubbles were not formed in the closed container. These findings indicate that the cavitation promoted the liquid penetration into wood.

The maximum volumetric swelling of the treated samples, $\beta_{\mathrm{m}}$, is shown in Fig. 8. For both species, the value of $\beta_{\mathrm{m}}$ for the semi-opened container was the same as that for the control, while the value for the closed container was slightly smaller than that for the control. This indicates that the wood sample was compressed in the closed container by the higher pressure at the liquid which surrounds the sample than the pressure inside the sample (Figs. 6 and 7). It was also indicated that the volume of wood was not so decreased in the semi-opened container. This may be due to the hydraulic pressure at the surrounding liquid experienced both higher and lower than the pressure inside the sample (Figs. 6 and 7). These findings indicate that the cavitation generation caused the liquid penetration into wood with less compressive deformation.

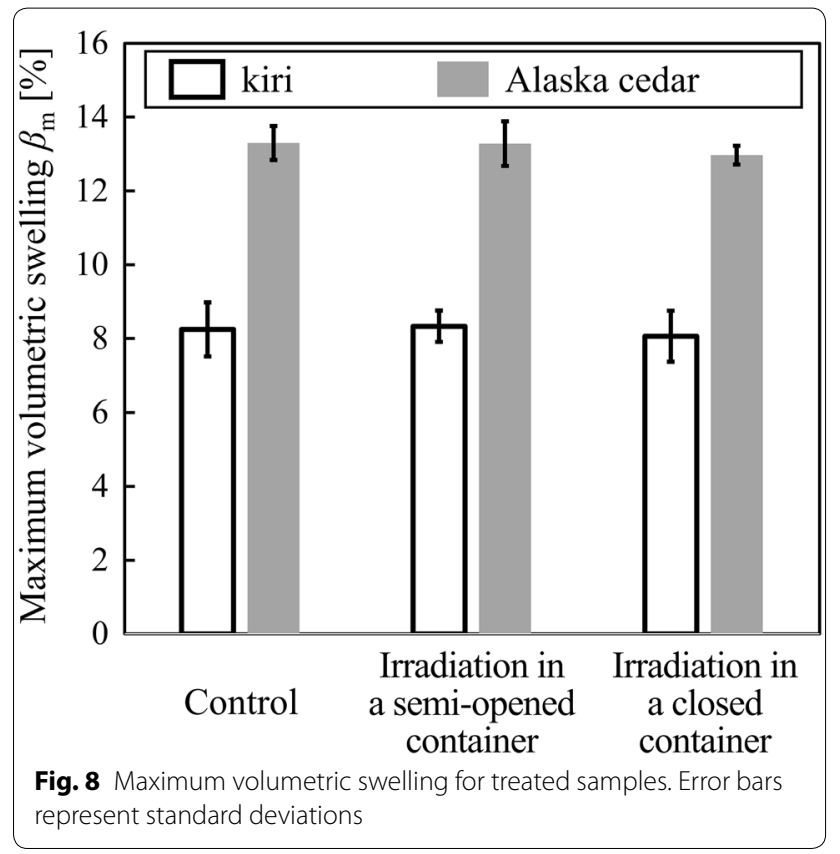

\section{Conclusion}

The purpose of this paper was to confirm the influence of the pulsive pressure waves on the liquid penetration into wood in the semi-opened container. The wood block samples of kiri and Alaska cedar were irradiated by the pulsive pressure waves in the closed or semi-opened container filled with liquid. The liquid penetration into the samples was promoted by the pressure-wave irradiation for both species. The degree of the penetration into kiri sample was higher than that into Alaska cedar one. There was little difference in the degree of the penetration between the closed and the semi-opened containers for both species. It was presumed from the measured pressure that the pressure-wave energy in the closed container was higher than that in the semi-opened container. It was also presumed that the cavitation generation was promoted in the semi-opened container. These findings indicate that the cavitation promoted the liquid penetration into wood. The compressive deformation during the pressure-wave irradiation was evaluated by comparing the maximum volumetric swelling for the irradiated sample with that for the non-irradiated one. The compressive deformation for the semi-opened container was slightly smaller than that for the closed container. This indicates that the pulsive pressure-wave irradiation in the semiopened container promoted the liquid penetration into wood with less compressive deformation.

It is practically required to improve the usability and safety of the apparatus with higher effect on the liquid penetration. Further studies are also necessary to clarify the physical behavior of the pressure-wave propagation 
in wood, to confirm the generation of the cavitation around the closed tissues in wood, and to investigate the distribution of compressive deformation after the wave irradiation. From the anatomical viewpoint, the closed tissues and cell walls are needed to be observed during or after the wave irradiation followed by the cavitation. It is necessary from the chemical viewpoint to investigate the influence of kinds of the liquid.

\section{Acknowledgements}

Part of this work was supported by JSPS KAKENHI Grant Number $16 \mathrm{H} 06895$ and by the research grant for Mission Research Fellows on Sustainable Humanosphere from Research Institute for Sustainable Humanosphere (RISH), Kyoto University.

\section{Authors' contributions}

ST has made substantial contributions to the conception, the design of the work, the acquisition, the analysis, and the interpretation of data, and has drafted the work. MS, TM, and KU have substantively revised the work. KK has made substantial contributions to the conception of the work. All authors read and approved the final manuscript.

\section{Funding}

No funding.

\section{Availability of data and materials}

Not applicable.

\section{Competing interests}

The authors declare that they have no competing interests.

\section{Author details}

${ }^{1}$ Research Institute for Sustainable Humanosphere, Kyoto University, Uji, Kyoto 611-0011, Japan. ${ }^{2}$ Structural Materials Research Institute, National Institute of Advanced Industrial Science and Technology, Nagoya, Aichi 463-8560, Japan.
Received: 12 July 2019 Accepted: 6 October 2019

Published online: 19 October 2019

\section{References}

1. Comstock GL, Côtè WA (1968) Factors affecting permeability and pit aspiration in coniferous sapwood. Wood Sci Technol 2:279-291

2. Imamura $Y$, Harada H, Saiki H (1974) Embedding substances of pit membranes in softwood tracheids and their degradation by enzymes. Wood Sci Technol 8:243-254

3. Durmaz S, Yildiz UC, Yildiz S (2015) Alkaline enzyme treatment of spruce wood to increase permeability. BioResources 10:4403-4410

4. Iida I, Yusuf S, Watanabe U, Imamura Y (2002) Liquid penetration of precompressed wood VII: the combined treatment of precompression and extraction in hot water on the liquid penetration of wood. J Wood Sci 48:81-85

5. Amemiya S, Siriban FR (1968) Research on wood preserving treatment (II) effect of dipping with supersonic waves on the treatment of wood. Bull Gov Forest Exp Station. 212:167-178

6. Nair HU, Simonsen J (1995) The pressure treatment of wood with sonic waves. Forest Prod J 45:59-64

7. Itoh S, Nagano S, Fujita M, Takano T, Honda R, Ikeda M (1998) The improvement of the permeability of wood by underwater shockwave. Metals Mater 4:843-846

8. Bolumara T, Bindrich U, Toepfl S, Toldrá F, Heinza V (2014) Effect of electrohydraulic shockwave treatment on tenderness, muscle cathepsin and peptidase activities and microstructure of beef loin steaks from Holstein young bulls. Meat Sci 98:759-765

9. The Japan society of mechanical engineers Ed. (2006) Fluid engineering. In: JSME mechanical engineer's handbooks, a4, pp 136-144

10. Siau JF (1984) Basic wood-moisture relationships. Transport processes in wood. Springer, Heidelberg, pp 1-34

\section{Publisher's Note}

Springer Nature remains neutral with regard to jurisdictional claims in published maps and institutional affiliations.

\section{Submit your manuscript to a SpringerOpen ${ }^{\odot}$ journal and benefit from:}

- Convenient online submission

- Rigorous peer review

- Open access: articles freely available online

- High visibility within the field

- Retaining the copyright to your article

Submit your next manuscript at springeropen.com 